\title{
Data-driven Identification of Reaction Network in Oxidative Coupling of Methane Reaction via Experimental Data
}

Itsuki Miyazato, * $, \uparrow, \S$ Shun Nishimura, $\uparrow$ Lauren Takahashi, $\uparrow, \S$ Junya Ohyama,, ,k and Keisuke Takahashi†, $\S$

$\dagger$ Department of Chemistry, Hokkaido University, Sapporo 060-8510, Japan. $\$$ Graduate School of Advanced Science and Technology, Japan Advanced Institute of Science and Technology, 1-1 Asahidai, Nomi, Ishikawa 923-1292 Japan qFaculty of Advanced Science and Technology, Kumamoto University, Kumamoto, 860-8555 Japan

$\S$ Center for Materials research by Information Integration $\left(\mathrm{CMI}^{2}\right)$, National Institute for Materials Science (NIMS), 1-2-1 Sengen, Tsukuba, Ibaraki 305-0047, Japan. kElements Strategy Initiative for Catalysts and Batteries (ESICB), Kyoto University, Katsura, Kyoto 615-8520 Japan

E-mail: miyazato@sci.hokudai.ac.jp 

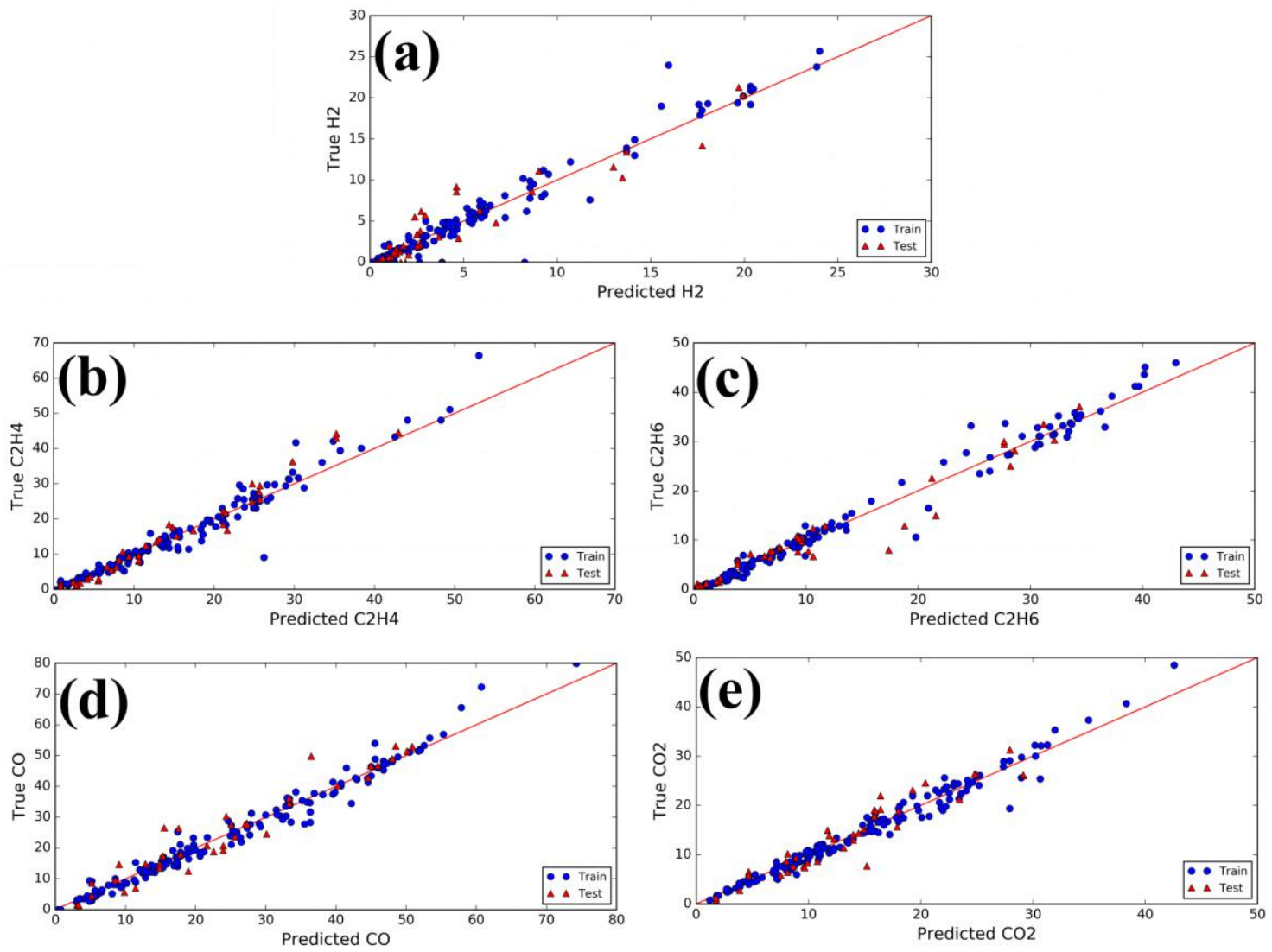

Figure S- 1. "True vs. Predicted" scatter plot in random forest regressors with different target(gas selectivities). 
(a) All range of $\mathrm{C} 2$ yield

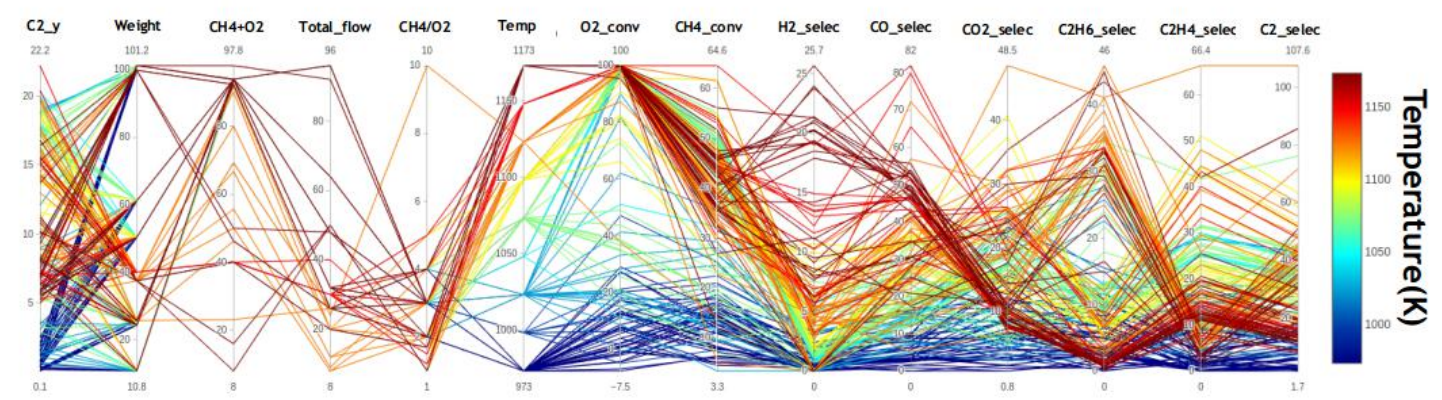

(b) C2 yield $<5$

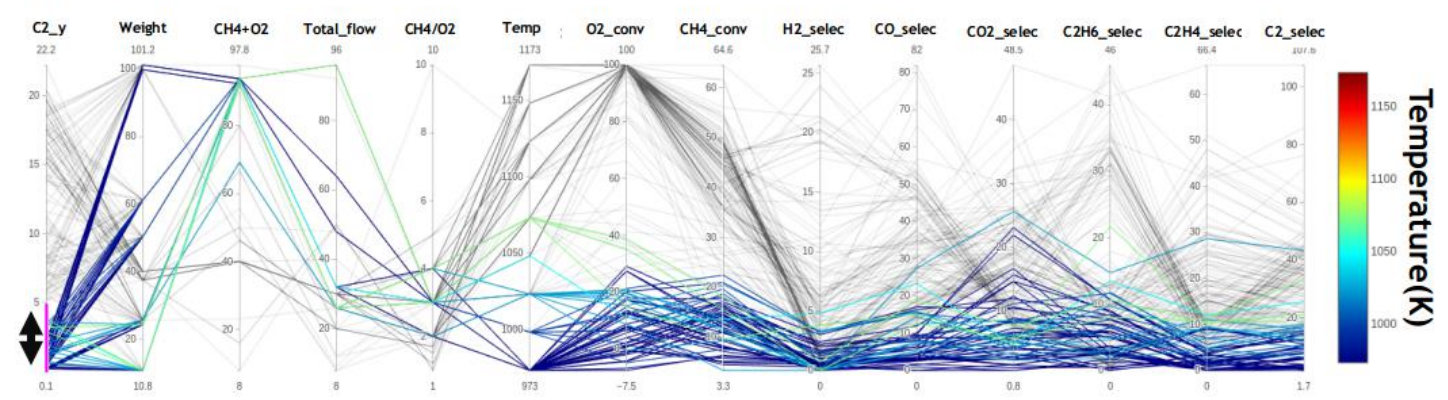

\section{(c) $15 \leq$ C2 yield}

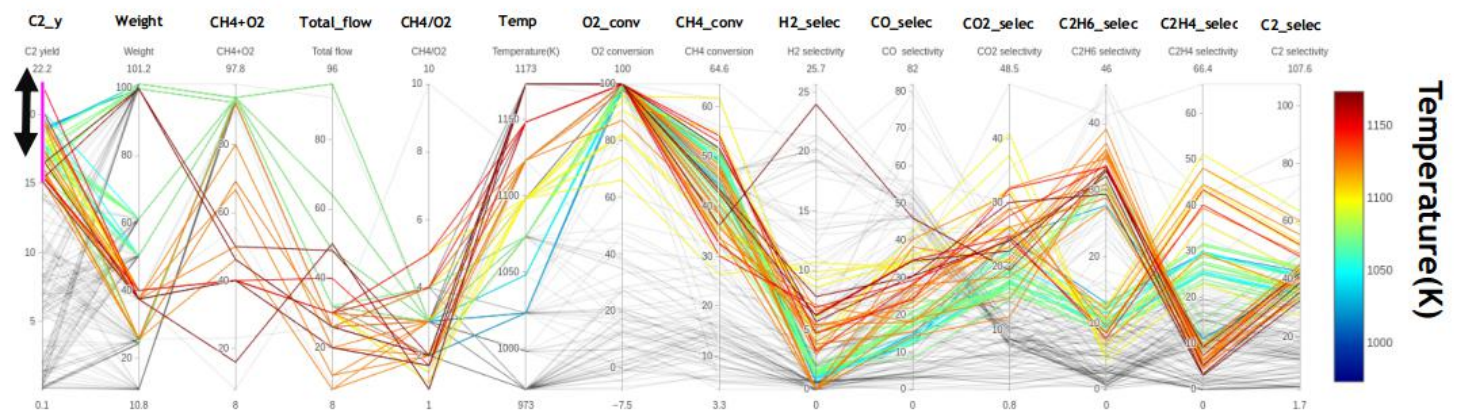

Figure S- 2. Parallel coordinates for all trained valuables against Temperature. (a)All range of C2 yield, (b) selected when $\mathrm{C} 2$ yield $<5$, and (c) selected when $\mathrm{C} 2$ yield $\geq 15$. 
Table S- 1. Data table of experimental conditions and reaction output. Note that data columns are explained in TABLE I. in main article. The original data has already reported in the previous study[1].

\begin{tabular}{|c|c|c|c|c|c|c|c|c|c|c|c|c|c|}
\hline \multirow{2}{*}{ No. } & \multicolumn{5}{|c|}{ Experimental Conditions } & \multicolumn{8}{|c|}{ Reaction output } \\
\hline & Weight & $\mathrm{CH} 4+\mathrm{O} 2$ & Total_flow & $\mathrm{CH} 4 \mathrm{O}_{2}$ & Temp & O2_conv & CH4_conv & $\mathrm{H} 2$ selec & CO selec & $\begin{array}{l}\mathrm{C} 2 \mathrm{H} 6 \\
\text { selec } \\
\end{array}$ & $\mathrm{CO} 2 \mathrm{selec}$ & $\begin{array}{l}\mathrm{C} 2 \mathrm{H} 4 \\
\text { selec } \\
\end{array}$ & $\mathrm{C} 2 \mathrm{y}$ \\
\hline 1 & 37.5 & 16 & 50 & 1 & 1123 & 100 & 4.9 & 0 & 31.7 & 41.2 & 48.5 & 66.4 & 5.2 \\
\hline 2 & 40.1 & 40 & 30 & 5 & 1098 & 66.2 & 26.3 & 10.7 & 32.4 & 12.4 & 21.2 & 51.1 & 16.7 \\
\hline 3 & 40.1 & 40 & 30 & 4 & 1098 & 74.4 & 30.1 & 9.9 & 34 & 10.5 & 23 & 48.1 & 17.6 \\
\hline 4 & 40.1 & 40 & 30 & 5 & 1123 & 100 & 32.8 & 5.4 & 22 & 12 & 25.1 & 48.1 & 19.7 \\
\hline 5 & 40.1 & 40 & 30 & 4 & 1123 & 100 & 35.9 & 4.8 & 24.6 & 10.8 & 26.3 & 44.6 & 19.9 \\
\hline 6 & 40.1 & 40.1 & 30 & 3 & 1098 & 81.8 & 35.3 & 9.2 & 36.2 & 8.6 & 26.2 & 44.3 & 18.7 \\
\hline 7 & 40.1 & 40 & 30 & 5 & 1148 & 100 & 30.1 & 6.9 & 29.3 & 8.5 & 24.1 & 43.4 & 15.6 \\
\hline 8 & 40.1 & 40.1 & 30 & 3 & 1098 & 82.6 & 36.2 & 8.6 & 34.6 & 8.4 & 26.1 & 43 & 18.6 \\
\hline 9 & 37.5 & 8 & 50 & 1 & 1173 & 100 & 11.9 & 0 & 27.8 & 43.6 & 35.3 & 42.1 & 10.2 \\
\hline 10 & 25.7 & 46.2 & 26 & 2 & 1073 & 20.1 & 6.9 & 0 & 33.5 & 34.6 & 23.8 & 41.7 & 5.3 \\
\hline 11 & 40.1 & 40 & 30 & 4 & 1148 & 100 & 32.5 & 6.3 & 34.7 & 7.7 & 24.7 & 40.1 & 15.5 \\
\hline 12 & 40.1 & 40.1 & 30 & 3 & 1123 & 100 & 40.5 & 4 & 28.4 & 9.4 & 29.1 & 39.4 & 19.7 \\
\hline 13 & 40.1 & 40.1 & 30 & 3 & 1148 & 100 & 34.8 & 6.3 & 42.8 & 6.8 & 26.4 & 36.3 & 15 \\
\hline 14 & 40.1 & 40.1 & 30 & 2 & 1098 & 90.9 & 46.4 & 6.1 & 35.4 & 6.3 & 32.2 & 36.1 & 19.7 \\
\hline 15 & 40.1 & 40.1 & 30 & 3 & 1148 & 100 & 35.9 & 5.7 & 41.3 & 6.4 & 25.9 & 33.3 & 14.3 \\
\hline 16 & 101.2 & 93.8 & 64 & 3 & 1073 & 100 & 41.7 & 1.1 & 19.4 & 12.9 & 16.6 & 31.7 & 18.5 \\
\hline 17 & 101.2 & 93.8 & 96 & 3 & 1073 & 100 & 39.1 & 1.4 & 23.5 & 12 & 15.2 & 31.4 & 17 \\
\hline 18 & 101.2 & 93.7 & 48 & 3 & 1073 & 100 & 42.2 & 1.3 & 18.8 & 12.6 & 18.3 & 31.2 & 18.5 \\
\hline
\end{tabular}




\begin{tabular}{|c|c|c|c|c|c|c|c|c|c|c|c|c|c|}
\hline 19 & 50.3 & 93.7 & 32 & 3 & 1048 & 98.4 & 49.4 & 1.6 & 14.8 & 10.5 & 19.1 & 30 & 20 \\
\hline 20 & 40.1 & 40.1 & 30 & 1.5 & 1098 & 93.9 & 54.8 & 4.4 & 38.1 & 5.1 & 37.3 & 29.8 & 19.1 \\
\hline 21 & 61.2 & 93.7 & 32 & 3 & 1048 & 100 & 49.4 & 0.9 & 13.1 & 11.4 & 20.6 & 29.7 & 20.3 \\
\hline 22 & 101.2 & 93.7 & 32 & 3 & 1023 & 98.1 & 44 & 1.2 & 12.2 & 13 & 24.4 & 29.6 & 18.8 \\
\hline 23 & 40.1 & 40.1 & 30 & 2 & 1123 & 100 & 47.5 & 2.9 & 40.3 & 7.2 & 31.3 & 29.5 & 17.5 \\
\hline 24 & 101.2 & 93.7 & 32 & 3 & 1048 & 100 & 45 & 1 & 14 & 12.3 & 22.5 & 29.4 & 18.7 \\
\hline 25 & 61.2 & 93.7 & 32 & 4 & 1073 & 100 & 44.2 & 1.5 & 12.4 & 11.6 & 17.1 & 28.9 & 17.9 \\
\hline 26 & 25.7 & 69.2 & 26 & 2 & 1023 & 8.8 & 3.3 & 0 & 27.4 & 14.7 & 25.6 & 28.6 & 1.4 \\
\hline 27 & 61.2 & 93.7 & 32 & 3 & 1073 & 100 & 48.2 & 1.2 & 17.5 & 10.3 & 18.9 & 27.7 & 18.3 \\
\hline 28 & 50.3 & 93.7 & 32 & 3 & 1073 & 100 & 48.9 & 1.4 & 18.6 & 9.7 & 17.3 & 27.3 & 18.1 \\
\hline 29 & 24.3 & 93.7 & 32 & 3 & 1073 & 98.6 & 48.7 & 2.1 & 17 & 9.1 & 17.6 & 27.2 & 17.6 \\
\hline 30 & 61.2 & 93.7 & 32 & 3 & 1073 & 100 & 48.6 & 1.3 & 16.9 & 10 & 19.4 & 26.1 & 17.5 \\
\hline 31 & 101.2 & 93.7 & 32 & 3 & 1073 & 100 & 43.3 & 1.6 & 21 & 10.6 & 19.9 & 26.1 & 15.9 \\
\hline 32 & 25.7 & 23.1 & 26 & 2 & 1123 & 37.7 & 14.1 & 0 & 30.8 & 46 & 19 & 25.8 & 10.1 \\
\hline 33 & 50.3 & 93.7 & 32 & 3 & 1048 & 99.3 & 50.9 & 1.1 & 13.6 & 9.4 & 17.3 & 25.6 & 17.8 \\
\hline 34 & 24.3 & 93.7 & 48 & 3 & 1073 & 43.8 & 23.4 & 6.2 & 26.4 & 12.9 & 7.7 & 25.6 & 9 \\
\hline 35 & 24.3 & 93.8 & 96 & 3 & 1173 & 95.4 & 35.5 & 8.3 & 42.6 & 4.9 & 7.6 & 25.5 & 10.8 \\
\hline 36 & 61.2 & 93.7 & 32 & 3 & 1048 & 100 & 51.6 & 0.9 & 12 & 9.3 & 17.5 & 25.2 & 17.8 \\
\hline 37 & 61.2 & 93.7 & 32 & 3 & 1073 & 100 & 51.3 & 1.2 & 13.7 & 8.5 & 16.7 & 25 & 17.2 \\
\hline 38 & 50.3 & 93.7 & 32 & 3 & 1048 & 99.7 & 50 & 1 & 13.5 & 9.7 & 17.8 & 24.9 & 17.3 \\
\hline 39 & 10.9 & 93.7 & 32 & 3 & 1098 & 80.3 & 36.2 & 8.1 & 36 & 5.8 & 10.5 & 24.1 & 10.8 \\
\hline 40 & 50.3 & 93.7 & 32 & 3 & 1073 & 100 & 49.9 & 1.3 & 17.4 & 8.5 & 16 & 23.9 & 16.2 \\
\hline
\end{tabular}




\begin{tabular}{|c|c|c|c|c|c|c|c|c|c|c|c|c|c|}
\hline 41 & 24.3 & 93.7 & 32 & 3 & 1098 & 99.4 & 47 & 2.4 & 23.4 & 7.8 & 15.9 & 23.4 & 14.6 \\
\hline 42 & 50.3 & 93.7 & 32 & 3 & 1073 & 100 & 48.9 & 1.4 & 17.5 & 8.6 & 16.5 & 23.1 & 15.5 \\
\hline 43 & 10.9 & 93.7 & 32 & 3 & 1123 & 96.9 & 40.1 & 6.3 & 37.3 & 5.4 & 11.8 & 23 & 11.4 \\
\hline 44 & 40.1 & 40 & 30 & 1.2 & 1098 & 95.7 & 61.8 & 3.3 & 42.3 & 4.2 & 40.7 & 23 & 16.8 \\
\hline 45 & 50.3 & 93.7 & 32 & 3 & 1098 & 100 & 46.2 & 2 & 28.1 & 7.7 & 14.1 & 22.3 & 13.9 \\
\hline 46 & 61.2 & 93.7 & 32 & 3 & 1098 & 100 & 45.5 & 2 & 27.5 & 7.8 & 15.2 & 21.9 & 13.6 \\
\hline 47 & 61.2 & 93.7 & 32 & 3 & 1098 & 100 & 49 & 1.8 & 21.1 & 7 & 14.4 & 21.3 & 13.8 \\
\hline 48 & 101.2 & 93.7 & 32 & 3 & 1098 & 100 & 40.1 & 3.3 & 36.3 & 6.8 & 14.5 & 20.6 & 11 \\
\hline 49 & 101.2 & 97.8 & 92 & 3 & 1173 & 100 & 35.5 & 17.9 & 51.9 & 2.1 & 9.8 & 20.6 & 8.1 \\
\hline 50 & 40.1 & 40.1 & 30 & 2 & 1148 & 100 & 43 & 5.4 & 65.6 & 3.7 & 21.4 & 19.8 & 10.1 \\
\hline 51 & 10.9 & 93.7 & 32 & 3 & 1073 & 36.8 & 22.9 & 6.8 & 31.3 & 9.9 & 6.2 & 19.6 & 6.7 \\
\hline 52 & 24.3 & 93.8 & 64 & 3 & 1173 & 100 & 36.3 & 7.6 & 47.5 & 3.2 & 8 & 19.6 & 8.3 \\
\hline 53 & 50.3 & 93.7 & 32 & 3 & 1098 & 100 & 47.3 & 1.9 & 26.5 & 6.7 & 13.2 & 19.5 & 12.4 \\
\hline 54 & 24.3 & 93.8 & 64 & 3 & 1073 & 25.6 & 16 & 4.9 & 19.9 & 17.9 & 6 & 19.3 & 5.9 \\
\hline 55 & 40.1 & 40.1 & 30 & 1.5 & 1123 & 100 & 54.1 & 2.6 & 56.9 & 5 & 29.8 & 19.1 & 13 \\
\hline 56 & 61.2 & 93.7 & 32 & 3 & 1023 & 41.4 & 29.2 & 2 & 14.9 & 12.4 & 13.2 & 18.5 & 9 \\
\hline 57 & 50.3 & 93.7 & 32 & 3 & 1098 & 100 & 46.3 & 2.1 & 27.6 & 6.5 & 13 & 18.5 & 11.6 \\
\hline 58 & 101.2 & 93.8 & 64 & 3 & 1173 & 100 & 37.8 & 21.1 & 51.7 & 1.4 & 10.2 & 18.5 & 7.5 \\
\hline 59 & 61.2 & 93.7 & 32 & 2 & 1073 & 100 & 55 & 1.3 & 28.8 & 7.3 & 22 & 18.4 & 14.2 \\
\hline 60 & 37.5 & 40 & 20 & 1.7 & 1073 & 34.8 & 19.4 & 2.7 & 33.9 & 31.5 & 25.4 & 17.9 & 9.6 \\
\hline 61 & 24.3 & 93.7 & 48 & 3 & 1173 & 100 & 37.3 & 14.2 & 53.1 & 1.7 & 7.6 & 17.7 & 7.2 \\
\hline 62 & 101.2 & 93.7 & 32 & 3 & 1123 & 100 & 39.3 & 10.2 & 48.9 & 2.4 & 10.3 & 17.3 & 7.7 \\
\hline
\end{tabular}




\begin{tabular}{|c|c|c|c|c|c|c|c|c|c|c|c|c|c|}
\hline 63 & 24.3 & 93.7 & 32 & 3 & 1123 & 100 & 44.3 & 3.9 & 37.8 & 4.4 & 9.9 & 17 & 9.5 \\
\hline 64 & 24.3 & 93.7 & 32 & 3 & 1048 & 51.1 & 32.2 & 5.7 & 26.6 & 7.7 & 8.3 & 16.8 & 7.9 \\
\hline 65 & 101.2 & 93.7 & 48 & 3 & 1173 & 100 & 38.6 & 23.8 & 53.3 & 1 & 11.3 & 16.8 & 6.9 \\
\hline 66 & 50.3 & 93.7 & 32 & 3 & 1123 & 100 & 43.7 & 5.1 & 41 & 3.6 & 9.4 & 16.7 & 8.9 \\
\hline 67 & 10.9 & 93.7 & 32 & 3 & 1148 & 98.7 & 38.6 & 10.3 & 51.5 & 2.3 & 7.4 & 16.7 & 7.4 \\
\hline 68 & 61.2 & 93.7 & 32 & 3 & 1123 & 100 & 43.4 & 6 & 41.4 & 3.2 & 9.9 & 16 & 8.3 \\
\hline 69 & 101.2 & 93.7 & 32 & 3 & 998 & 47.2 & 25.1 & 1 & 13 & 15.5 & 23.3 & 15.9 & 7.9 \\
\hline 70 & 25.7 & 69.2 & 26 & 2 & 1073 & 38.2 & 18.6 & 0 & 35.3 & 29.5 & 14.1 & 15.6 & 8.4 \\
\hline 71 & 101.2 & 93.7 & 32 & 3 & 1148 & 100 & 40.9 & 19.3 & 51.1 & 1.2 & 11.3 & 15.3 & 6.7 \\
\hline 72 & 61.2 & 93.7 & 32 & 3 & 1123 & 100 & 45.8 & 5.2 & 37.5 & 3.1 & 8.6 & 15.2 & 8.4 \\
\hline 73 & 50.3 & 93.7 & 32 & 3 & 1023 & 62.1 & 35.5 & 5 & 25.3 & 6.8 & 10.6 & 15.2 & 7.8 \\
\hline 74 & 50.3 & 93.7 & 32 & 3 & 1123 & 100 & 44.7 & 5 & 40.2 & 3.1 & 8.7 & 15.2 & 8.2 \\
\hline 75 & 24.3 & 93.7 & 32 & 3 & 1148 & 100 & 44.5 & 11.6 & 46.7 & 1.5 & 7.7 & 15.2 & 7.4 \\
\hline 76 & 99.8 & 92.3 & 26 & 4 & 1073 & 99.9 & 34 & 1.9 & 8.5 & 33 & 14.8 & 15 & 16.3 \\
\hline 77 & 50.3 & 93.7 & 32 & 3 & 1123 & 100 & 44.5 & 5.8 & 41 & 2.9 & 8 & 14.9 & 7.9 \\
\hline 78 & 10.9 & 93.7 & 32 & 3 & 1173 & 100 & 40.5 & 18.5 & 55.7 & 1 & 6.9 & 14.9 & 6.4 \\
\hline 79 & 50.3 & 93.7 & 32 & 3 & 1023 & 26.7 & 24.5 & 2.1 & 14.7 & 12.9 & 10.2 & 14.7 & 6.8 \\
\hline 80 & 37.5 & 91.7 & 24 & 10 & 1123 & 99 & 22.7 & 4.7 & 5.2 & 31.3 & 6.9 & 14.5 & 10.4 \\
\hline 81 & 50.3 & 93.7 & 32 & 3 & 1148 & 100 & 44.6 & 13.4 & 46.8 & 1.3 & 8.7 & 14.4 & 7 \\
\hline 82 & 61.2 & 93.7 & 32 & 3 & 1148 & 100 & 44.4 & 14.9 & 46.4 & 1.3 & 9.8 & 14.2 & 6.9 \\
\hline 83 & 61.2 & 93.7 & 32 & 4 & 1173 & 100 & 40.8 & 19.4 & 42.7 & 0.8 & 8.8 & 13.8 & 6 \\
\hline 84 & 99.8 & 92.3 & 26 & 4 & 1023 & 81.7 & 26.5 & 3.8 & 14.7 & 22.6 & 15.7 & 13.7 & 9.6 \\
\hline
\end{tabular}




\begin{tabular}{|c|c|c|c|c|c|c|c|c|c|c|c|c|c|}
\hline 85 & 50.3 & 93.7 & 32 & 3 & 1148 & 100 & 45.4 & 13.5 & 46.1 & 1.1 & 8.1 & 13.4 & 6.6 \\
\hline 86 & 24.3 & 93.7 & 32 & 3 & 1173 & 100 & 45.9 & 19.2 & 49.6 & 0.7 & 8.1 & 13.4 & 6.5 \\
\hline 87 & 50.3 & 93.7 & 32 & 3 & 1148 & 100 & 45.4 & 13.9 & 46 & 1.1 & 7.8 & 13 & 6.4 \\
\hline 88 & 101.2 & 93.7 & 32 & 3 & 1173 & 100 & 41.9 & 25.7 & 51.5 & 0.6 & 13.4 & 12.9 & 5.7 \\
\hline 89 & 61.2 & 93.7 & 32 & 3 & 1148 & 100 & 46.4 & 13 & 43.4 & 1.1 & 7.9 & 12.6 & 6.4 \\
\hline 90 & 50.3 & 93.7 & 32 & 3 & 1173 & 100 & 45.8 & 20.3 & 49 & 0.7 & 9.4 & 12.5 & 6 \\
\hline 91 & 61.2 & 93.7 & 32 & 3 & 1173 & 100 & 45.5 & 21.4 & 48.2 & 0.7 & 10.9 & 12.3 & 5.9 \\
\hline 92 & 99.8 & 92.3 & 26 & 3 & 1073 & 99.9 & 40 & 1.6 & 12.1 & 31.1 & 17.6 & 12.2 & 17.4 \\
\hline 93 & 50.3 & 93.7 & 32 & 3 & 1023 & 28.4 & 26.8 & 2.1 & 14 & 9.9 & 8.6 & 12.1 & 5.9 \\
\hline 94 & 10.9 & 93.7 & 32 & 3 & 1048 & 11 & 13.6 & 4.8 & 23.3 & 13.5 & 4.8 & 12 & 3.5 \\
\hline 95 & 40.1 & 40.1 & 30 & 1.5 & 1148 & 100 & 53.6 & 12.2 & 79.9 & 1.2 & 17.8 & 11.9 & 7 \\
\hline 96 & 61.2 & 93.7 & 32 & 3 & 1173 & 100 & 46.2 & 20.9 & 47 & 0.6 & 10.5 & 11.8 & 5.7 \\
\hline 97 & 61.2 & 93.7 & 32 & 3 & 1023 & 33.3 & 28.1 & 1.5 & 10.4 & 10.2 & 11.3 & 11.7 & 6.2 \\
\hline 98 & 50.3 & 93.7 & 32 & 3 & 1173 & 100 & 46.5 & 20.2 & 48.5 & 0.6 & 9 & 11.5 & 5.6 \\
\hline 99 & 24.3 & 93.8 & 96 & 3 & 1073 & 14.8 & 9.6 & 3.8 & 15.1 & 21.7 & 5 & 11.4 & 3.2 \\
\hline 100 & 99.8 & 92.3 & 26 & 3 & 1023 & 90 & 32.7 & 4.1 & 19.2 & 25.8 & 19 & 11.2 & 12.1 \\
\hline 101 & 50.3 & 93.7 & 32 & 3 & 1173 & 100 & 46.5 & 20.2 & 48.1 & 0.6 & 8.7 & 11.2 & 5.5 \\
\hline 102 & 40.1 & 40 & 30 & 1.2 & 1123 & 100 & 61.5 & 4.1 & 72.3 & 2.6 & 25.6 & 11.2 & 8.5 \\
\hline 103 & 99.8 & 92.3 & 26 & 2 & 1023 & 99.8 & 49.2 & 0.6 & 14.8 & 27.7 & 24.5 & 11 & 19 \\
\hline 104 & 25.7 & 92.3 & 26 & 2 & 1023 & 21 & 13 & 0 & 12.4 & 4.9 & 8.6 & 11 & 2.1 \\
\hline 105 & 61.2 & 93.7 & 32 & 3 & 1173 & 100 & 47.5 & 19.2 & 45.3 & 0.1 & 8.8 & 11 & 5.3 \\
\hline 106 & 25.7 & 92.3 & 26 & 4 & 1073 & 73 & 24.4 & 7.5 & 21.1 & 24 & 11.5 & 10.8 & 8.5 \\
\hline
\end{tabular}




\begin{tabular}{|c|c|c|c|c|c|c|c|c|c|c|c|c|c|}
\hline 107 & 10.8 & 92.3 & 26 & 4 & 1073 & 35.2 & 15.5 & 0 & 12.5 & 8 & 5.8 & 10.7 & 2.9 \\
\hline 108 & 10.8 & 92.3 & 26 & 3 & 1073 & 38.8 & 18 & 0.7 & 18.8 & 10.6 & 7.6 & 10.4 & 3.8 \\
\hline 109 & 37.5 & 90 & 20 & 5 & 1123 & 99.7 & 31.8 & 4.3 & 9.7 & 33.8 & 11.8 & 10.1 & 14 \\
\hline 110 & 25.7 & 92.3 & 26 & 3 & 1073 & 96.4 & 37.4 & 4.9 & 17.1 & 32.8 & 17 & 10 & 16 \\
\hline 111 & 25.7 & 92.3 & 26 & 2 & 1073 & 99.3 & 49 & 0 & 19.2 & 30 & 22 & 9.7 & 19.5 \\
\hline 112 & 25.7 & 92.3 & 26 & 4 & 1023 & 17.3 & 9.7 & 0 & 5.2 & 2.2 & 5.4 & 9.5 & 1.1 \\
\hline 113 & 25.7 & 92.3 & 26 & 4 & 1123 & 99.8 & 34.2 & 0 & 14.3 & 35.2 & 12.1 & 9.5 & 15.3 \\
\hline 114 & 10.8 & 92.3 & 26 & 2 & 1073 & 49.1 & 25.3 & 1.3 & 30.3 & 12.9 & 10.2 & 9.4 & 5.7 \\
\hline 115 & 99.8 & 92.3 & 26 & 3 & 973 & 27.5 & 12.6 & 1.7 & 7.9 & 2.6 & 14.7 & 9.3 & 1.5 \\
\hline 116 & 25.7 & 92.3 & 26 & 3 & 1023 & 19.9 & 11.2 & 0 & 7.2 & 3 & 6.3 & 9.3 & 1.4 \\
\hline 117 & 37.5 & 80 & 20 & 3 & 1123 & 99.8 & 38.4 & 3.8 & 15.4 & 35.4 & 19.9 & 9.3 & 17.2 \\
\hline 118 & 99.8 & 92.3 & 26 & 2 & 1073 & 99.9 & 48.7 & 1.4 & 18.5 & 27.3 & 22.6 & 9.2 & 17.8 \\
\hline 119 & 10.8 & 92.3 & 26 & 2 & 1023 & 20.7 & 13.9 & 0 & 14.4 & 4.6 & 4.6 & 9.2 & 1.9 \\
\hline 120 & 99.8 & 92.3 & 26 & 4 & 973 & 27.4 & 11.9 & 1 & 5.7 & 1.8 & 12.4 & 9.1 & 1.3 \\
\hline 121 & 99.8 & 92.3 & 26 & 4 & 1123 & 99.9 & 33.2 & 4.6 & 12.2 & 33.2 & 13.5 & 9.1 & 14 \\
\hline 122 & 37.5 & 16 & 50 & 1 & 1173 & 100 & 36.2 & 24 & 46 & 32.9 & 19.4 & 9.1 & 15.2 \\
\hline 123 & 99.8 & 92.3 & 26 & 2 & 973 & 29.1 & 15.6 & 2.4 & 11.9 & 3.3 & 16.7 & 8.9 & 1.9 \\
\hline 124 & 61.2 & 93.7 & 32 & 2 & 1173 & 100 & 56.1 & 21.3 & 52.9 & 0.4 & 13.9 & 8.4 & 5 \\
\hline 125 & 25.7 & 92.3 & 26 & 3 & 1123 & 99.9 & 39 & 0 & 18.9 & 33.5 & 15 & 8.4 & 16.3 \\
\hline 126 & 25.7 & 92.3 & 26 & 2 & 1073 & 98.5 & 50.3 & 0 & 20.8 & 29.4 & 19.2 & 8 & 18.8 \\
\hline 127 & 24.3 & 93.7 & 32 & 3 & 1023 & 15.3 & 20.5 & 3.3 & 15.7 & 9.7 & 6 & 8 & 3.6 \\
\hline 128 & 40.1 & 40 & 30 & 1.2 & 1148 & 100 & 64.6 & 19 & 82 & 0.5 & 19.8 & 7.7 & 5.3 \\
\hline
\end{tabular}




\begin{tabular}{|c|c|c|c|c|c|c|c|c|c|c|c|c|c|}
\hline 129 & 99.8 & 92.3 & 26 & 3 & 1123 & 99.9 & 38.7 & 3.8 & 16.4 & 31.1 & 16 & 7.6 & 15 \\
\hline 130 & 25.7 & 69.2 & 26 & 2 & 1123 & 99.8 & 45.7 & 0 & 26.9 & 35.8 & 23.1 & 7.3 & 19.7 \\
\hline 131 & 37.5 & 40 & 40 & 1.7 & 1148 & 99.8 & 54.2 & 3.2 & 28.3 & 33.7 & 27.9 & 7.3 & 22.2 \\
\hline 132 & 37.5 & 55.6 & 9 & 4 & 1123 & 99.7 & 29.1 & 0 & 14.5 & 41.2 & 19.5 & 7.2 & 14.1 \\
\hline 133 & 50.3 & 93.7 & 32 & 3 & 998 & 21.3 & 22.4 & 3.2 & 15.3 & 8.4 & 8.2 & 7 & 3.5 \\
\hline 134 & 10.8 & 92.3 & 26 & 3 & 1023 & 19.5 & 11.7 & 0 & 7.7 & 2.9 & 3.3 & 6.9 & 1.1 \\
\hline 135 & 37.5 & 66.7 & 12 & 3 & 1123 & 99.8 & 35.9 & 5.5 & 18 & 37.1 & 21.2 & 6.4 & 15.6 \\
\hline 136 & 25.7 & 92.3 & 26 & 2 & 1123 & 99.9 & 48.3 & 0 & 27.9 & 27.4 & 18.4 & 6.3 & 16.3 \\
\hline 137 & 37.5 & 80 & 20 & 2 & 1123 & 99.8 & 48.3 & 3.4 & 23.6 & 30.3 & 24.6 & 6.3 & 17.7 \\
\hline 138 & 99.8 & 46.2 & 26 & 4 & 1173 & 99.8 & 26.9 & 11.2 & 17.4 & 45.1 & 17.6 & 5.9 & 13.7 \\
\hline 139 & 25.7 & 46.2 & 26 & 2 & 1123 & 87.3 & 38.4 & 0 & 38.2 & 36.2 & 20.6 & 5.9 & 16.1 \\
\hline 140 & 37.5 & 50 & 8 & 3 & 1123 & 99.8 & 33.7 & 7.1 & 18 & 39.2 & 24.1 & 5.9 & 15.2 \\
\hline 141 & 61.2 & 93.7 & 32 & 3 & 998 & 12.8 & 18.1 & 0.9 & 8.8 & 11.6 & 11.2 & 5.6 & 3.1 \\
\hline 142 & 99.8 & 92.3 & 26 & 2 & 1123 & 99.9 & 47.6 & 3.1 & 27.8 & 25 & 18.9 & 5.5 & 14.5 \\
\hline 143 & 37.5 & 40 & 20 & 1.7 & 1123 & 97.6 & 50.9 & 6.6 & 27.7 & 34.8 & 32.3 & 5.4 & 20.4 \\
\hline 144 & 99.8 & 50 & 48 & 2 & 1173 & 99.8 & 42.6 & 6.2 & 34.5 & 33.2 & 22.4 & 5.2 & 16.4 \\
\hline 145 & 37.5 & 40 & 20 & 1.7 & 1148 & 99.8 & 53 & 4.7 & 24.2 & 33.5 & 32.1 & 5.1 & 20.4 \\
\hline 146 & 37.5 & 66.7 & 12 & 2 & 1123 & 99.8 & 47 & 0 & 25.6 & 28.8 & 26.1 & 4.8 & 15.8 \\
\hline 147 & 10.9 & 93.7 & 32 & 3 & 1023 & -0.5 & 9.2 & 3.2 & 15.9 & 11.8 & 4 & 4.8 & 1.5 \\
\hline 148 & 61.2 & 93.7 & 32 & 2 & 973 & 20.2 & 20.9 & 3.2 & 17 & 7.7 & 10.5 & 4.7 & 2.6 \\
\hline 149 & 10.8 & 92.3 & 26 & 4 & 1023 & 18.5 & 11.7 & 0 & 4.7 & 1.8 & 2.4 & 4.6 & 0.7 \\
\hline 150 & 50.3 & 93.7 & 32 & 3 & 998 & 6.5 & 16.7 & 1.2 & 8.9 & 10.6 & 8.3 & 4.6 & 2.5 \\
\hline
\end{tabular}




\begin{tabular}{|c|c|c|c|c|c|c|c|c|c|c|c|c|c|}
\hline 151 & 37.5 & 50 & 8 & 2 & 1123 & 99.8 & 43.2 & 5.2 & 24 & 30.9 & 28.9 & 4.4 & 15.2 \\
\hline 152 & 61.2 & 93.7 & 32 & 2 & 973 & 18 & 17.6 & 3 & 16.3 & 8.3 & 12 & 4.2 & 2.2 \\
\hline 153 & 101.2 & 93.7 & 32 & 3 & 973 & 17.3 & 14.2 & 0.6 & 10 & 10.7 & 21.9 & 3.8 & 2.1 \\
\hline 154 & 25.7 & 92.3 & 26 & 2 & 973 & 13.6 & 9.2 & 0 & 4.5 & 0.3 & 4.5 & 3.5 & 0.3 \\
\hline 155 & 25.7 & 92.3 & 26 & 4 & 1173 & 99.8 & 32.5 & 0 & 25.1 & 31.1 & 9.5 & 3.5 & 11.2 \\
\hline 156 & 101.2 & 93.7 & 32 & 3 & 973 & 17.2 & 13.6 & 0.5 & 9.6 & 10.6 & 23.1 & 3.5 & 1.9 \\
\hline 157 & 99.8 & 46.2 & 26 & 2 & 1173 & 99.8 & 43.4 & 7.8 & 30.4 & 32.1 & 24 & 3.3 & 15.3 \\
\hline 158 & 25.7 & 92.3 & 26 & 3 & 1173 & 99.9 & 36.5 & 9.5 & 34.9 & 26.8 & 10.6 & 3.3 & 11 \\
\hline 159 & 10.8 & 92.3 & 26 & 2 & 973 & 14.9 & 10.8 & 0.5 & 4.2 & 0.4 & 1.6 & 3.3 & 0.4 \\
\hline 160 & 37.5 & 40 & 20 & 1.7 & 1173 & 99.8 & 51.7 & 5.7 & 30.4 & 29.4 & 30 & 3 & 16.8 \\
\hline 161 & 50.3 & 93.7 & 32 & 3 & 998 & 6.4 & 18.7 & 0.9 & 7 & 7.6 & 6.5 & 3 & 2 \\
\hline 162 & 99.8 & 92.3 & 26 & 4 & 1173 & 99.9 & 31.4 & 8.6 & 20.8 & 28.1 & 11.4 & 2.9 & 9.7 \\
\hline 163 & 61.2 & 93.7 & 32 & 3 & 973 & 8.8 & 17.1 & 2 & 9.4 & 6.9 & 8.3 & 2.5 & 1.6 \\
\hline 164 & 61.2 & 93.7 & 32 & 3 & 998 & 11.8 & 19.6 & 0.6 & 5.7 & 6.7 & 8.7 & 2.5 & 1.8 \\
\hline 165 & 99.8 & 92.3 & 26 & 3 & 1173 & 99.9 & 35.9 & 8 & 30.7 & 23.5 & 12.7 & 2.4 & 9.3 \\
\hline 166 & 25.7 & 92.3 & 26 & 3 & 973 & 12.6 & 8.2 & 0 & 2.5 & 0 & 3.5 & 2.4 & 0.2 \\
\hline 167 & 10.8 & 92.3 & 26 & 3 & 973 & 12.9 & 9.6 & 0 & 1.7 & 0.5 & 1.2 & 2.2 & 0.3 \\
\hline 168 & 24.3 & 93.7 & 32 & 3 & 998 & 2 & 15.2 & 1.6 & 8.1 & 6.4 & 4.2 & 2.1 & 1.3 \\
\hline 169 & 25.7 & 92.3 & 26 & 2 & 1173 & 99.9 & 43.7 & 9.1 & 54 & 16.5 & 10.9 & 2 & 8.1 \\
\hline 170 & 50.3 & 93.7 & 32 & 3 & 973 & 5.7 & 17.1 & 2.2 & 9.2 & 5.8 & 5.4 & 2 & 1.3 \\
\hline 171 & 25.7 & 92.3 & 26 & 4 & 973 & 11.4 & 8.1 & 0 & 1.5 & 0.1 & 2.8 & 1.9 & 0.2 \\
\hline 172 & 50.3 & 93.7 & 32 & 3 & 973 & 4 & 14.9 & 2 & 8.6 & 5.8 & 6.1 & 1.7 & 1.1 \\
\hline
\end{tabular}




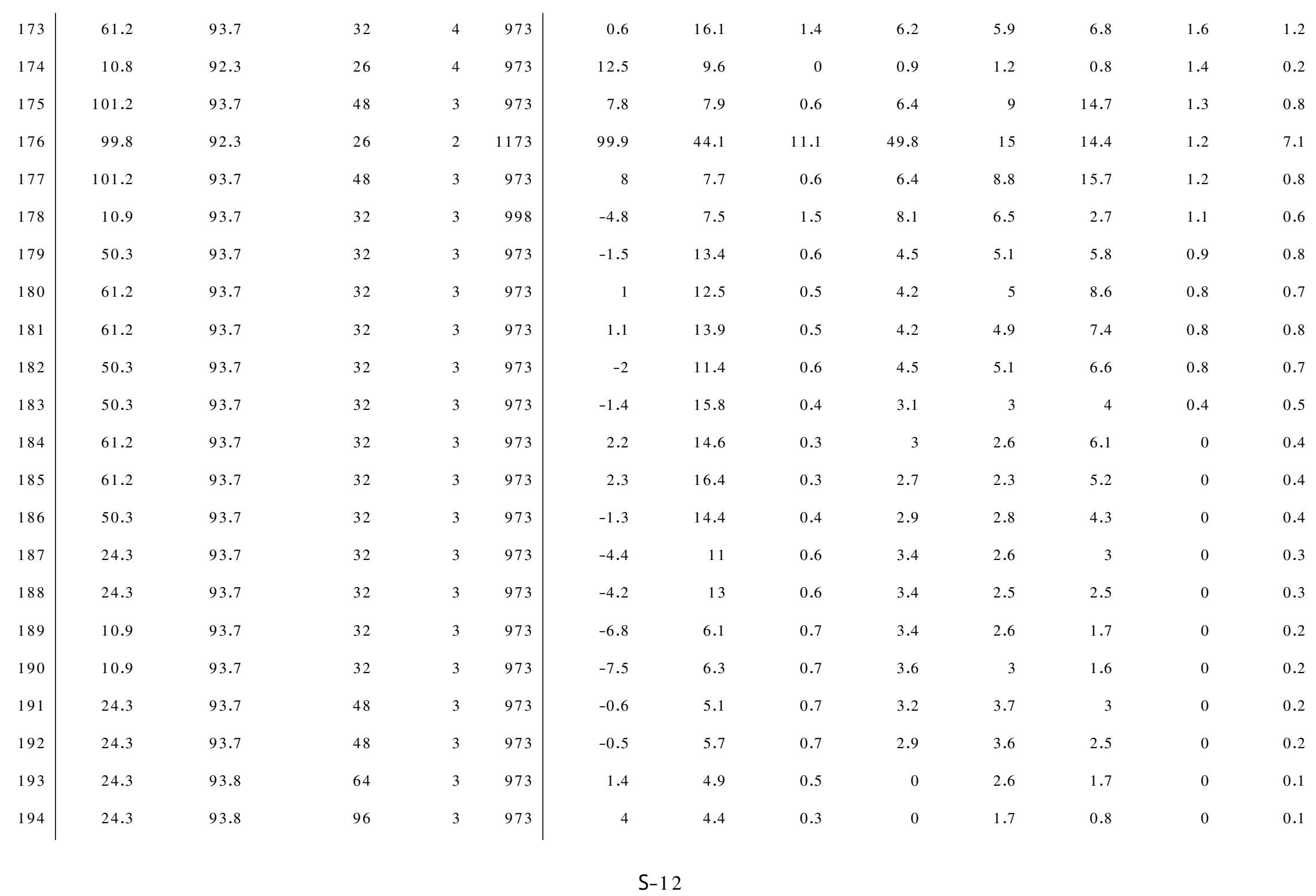




\begin{tabular}{l|lllll|llllllll}
195 & 101.2 & 93.8 & 64 & 3 & 973 & 7.5 & 7.1 & 0.5 & 4.5 & 6.5 & 10.7 & 0 & 0.5 \\
196 & 101.2 & 93.8 & 96 & 3 & 973 & 6.9 & 4.8 & 0.5 & 3.5 & 5.5 & 9 & 0 & 0.3 \\
\hline \hline
\end{tabular}


Table S- 2. Cross validation (CV) result against target selectivity by using experimental condition + X. Note that Avg. Med. And Std. indicates average score, median and standard deviation of $\mathrm{CV}$ scores in 10 random states.

\begin{tabular}{|c|c|c|c|c|}
\hline $\begin{array}{c}\text { Target } \\
\text { selectivity }\end{array}$ & $x$ & Avg. & Med. & Std. \\
\hline \multirow[t]{5}{*}{$\mathrm{H}_{2}$} & (None) & 0.71 & 0.67 & 0.14 \\
\hline & $\mathrm{CO}$ & 0.73 & 0.74 & 0.14 \\
\hline & $\mathrm{C}_{2} \mathrm{H}_{6}$ & 0.74 & 0.75 & 0.14 \\
\hline & $\mathrm{CO}_{2}$ & 0.80 & 0.79 & 0.09 \\
\hline & $\mathrm{C}_{2} \mathrm{H}_{4}$ & 0.73 & 0.70 & 0.12 \\
\hline \multirow[t]{5}{*}{$\mathrm{CO}$} & (None) & 0.80 & 0.83 & 0.12 \\
\hline & $\mathrm{H}_{2}$ & 0.80 & 0.80 & 0.08 \\
\hline & $\mathrm{C}_{2} \mathrm{H}_{6}$ & 0.90 & 0.91 & 0.02 \\
\hline & $\mathrm{CO}_{2}$ & 0.78 & 0.82 & 0.12 \\
\hline & $\mathrm{C}_{2} \mathrm{H}_{4}$ & 0.84 & 0.87 & 0.09 \\
\hline \multirow[t]{5}{*}{$\mathrm{C}_{2} \mathrm{H}_{6}$} & (None) & 0.83 & 0.91 & 0.12 \\
\hline & $\mathrm{H}_{2}$ & 0.80 & 0.82 & 0.09 \\
\hline & $\mathrm{CO}$ & 0.84 & 0.87 & 0.07 \\
\hline & $\mathrm{CO}_{2}$ & 0.78 & 0.79 & 0.11 \\
\hline & $\mathrm{C}_{2} \mathrm{H}_{4}$ & 0.82 & 0.88 & 0.12 \\
\hline \multirow[t]{5}{*}{$\mathrm{CO}_{2}$} & (None) & 0.77 & 0.76 & 0.06 \\
\hline & $\mathrm{H}_{2}$ & 0.79 & 0.78 & 0.06 \\
\hline & $\mathrm{CO}$ & 0.78 & 0.78 & 0.09 \\
\hline & $\mathrm{C}_{2} \mathrm{H}_{6}$ & 0.78 & 0.80 & 0.07 \\
\hline & $\mathrm{C}_{2} \mathrm{H}_{4}$ & 0.85 & 0.85 & 0.04 \\
\hline \multirow[t]{5}{*}{$\mathrm{C}_{2} \mathrm{H}_{4}$} & (None) & 0.77 & 0.77 & 0.10 \\
\hline & $\mathrm{H}_{2}$ & 0.80 & 0.79 & 0.06 \\
\hline & $\mathrm{CO}$ & 0.76 & 0.74 & 0.07 \\
\hline & $\mathrm{C}_{2} \mathrm{H}_{6}$ & 0.80 & 0.79 & 0.07 \\
\hline & $\mathrm{CO}_{2}$ & 0.83 & 0.84 & 0.08 \\
\hline
\end{tabular}

Reference

[1] Ohyama, J.; Nishimura, S.; Takahashi, K. Data Driven Determination of Reaction Conditions in Oxidative Coupling of Methane via Machine Learning. ChemCatChem 2019, 11, 4307-4313. 